https://doi.org/10.7559/gestaoedesenvolvimento.2019.386

Data de receção: 26/03/2019 Data de aceitação: 09/07/2019

\title{
POTENCIAR A ATENÇÃO/CONCENTRAÇÃO PELO REHACOG
}

\section{ENHANCING ATTENTION / CONCENTRATION BY REHACOG}

\section{Ana Paula Couceiro Figueira ${ }^{1}$ orcid.org/0000-0001-5998-3046 \\ Filipa Rocha ${ }^{2}$}

Resumo: $O$ constructo atenção/concentração é um domínio complexo de análise, mas tem-se revelado de grande importância no desempenho académico e mesmo em tarefas da vida diária.

Explorando as potencialidades de alguns módulos do programa de intervenção neuropsicológica REHACOG, foi realizado um estudo descritivo e exploratório com 37 crianças/adolescentes, do sexo feminino e masculino, com idades compreendidas entre os 7 e os 17 anos, com fragilidades atencionais. A amostra foi dividida e analisada em função de ciclos de escolaridade. Trata-se de um estudo de pré e pós-teste e a intervenção de algumas tarefas dos módulos de atenção do REHACOG. Os resultados obtidos revelam algumas diferenças nos diferentes ciclos de estudo, sugerindo relações positivas $e$ estatisticamente significativas entre as variáveis sob análise.

Palavras-chave: Atenção; REHACOG; Funções Executivas

\begin{abstract}
In the last decades, several studies in the field of Psychology have examined information on different occurrences related to the attention construct. In order to verify the potential of some modules of REHACOG intervention program, we performed a descriptive and exploratory study with 37 children and adolescents, female and male, with attentional weaknesses, aged between 7 and 17
\end{abstract}

${ }^{1}$ Faculdade de Psicologia e de Ciências da Educação, da Universidade de Coimbra. Email: apcouceirofigueira@gmail.com

${ }^{2}$ Faculdade de Psicologia e de Ciências da Educação, da Universidade de Coimbra 
years. The sample was divided and analysed in terms of levels of studies. It is a pre and post-testing study, applying tasks from REHACOG. The results uncover some differences and suggest positive and statistically significant relationships between the variables.

Keywords: Attention; REHACOG; Executive functions

\section{INTRODUÇÃO}

Historicamente, o pensamento dominante na psicologia foi sobretudo direcionado para o estudo dos aspetos "anormais" ou desviantes do comportamento humano. Segundo Seligman (2002), o enfoque era colocado nas emoções negativas e as experiências que traziam felicidade e bem-estar passavam despercebidas. Nas duas últimas décadas, assistimos a uma mudança gradual de paradigma, imposta pela psicologia positiva. Neste contexto, a psicologia deverá passar pela identificação e estimulação dos atributos e qualidades das pessoas, como forma de complemento e ajuda na compreensão dos seus problemas, não se limitando apenas ao tratamento de patologias (Seligman, 2002).

No âmbito da atenção, a aprendizagem interliga-se com os conceitos de atenção e de concentração, sendo imprescindível tratar de um conceito sem referir o outro (Rebelo, 1994). Podemos verificar esta questão perante os professores que se queixam frequentemente do facto de os seus alunos não prestarem atenção e andarem distraídos. A distração e a falta de concentração são os motivos mais indicados, quer em contexto de aprendizagem, quer de comportamento, etc. É consensual a ideia de que a atenção e a concentração são conceitos importantes para codificar, memorizar e assimilar informações, tornando-se necessário conhecê-los e avaliá-los, mais especificamente na sua relação com o ensino e a aprendizagem, de modo a, por um lado, saber os mecanismos que a determinam e, por outro, potenciá-los para utilizar estratégias adequadas de intervenção com alunos que manifestam fragilidades (Rebelo, 1994). A educação é um dos aspetos mais importantes do desenvolvimento dos recursos pessoais a que todas as crianças têm direito (Karande \& Kulkarni, 2005). É na escola que as crianças têm a oportunidade de se desenvolverem cognitiva, social e afetivamente. 
Assumindo este contexto, o objetivo do presente trabalho é verificar os ganhos, ao nível da atenção, face à aplicação de algumas tarefas do módulo de atenção do programa de intervenção neuropsicológica REHACOG (Figueira \& Paixão, in press). Foi realizado um estudo descritivo e exploratório com 37 crianças e adolescentes com fragilidades atencionais, com idades compreendidas entre os 7 e os 17 anos. Trata-se de um estudo com pré e pós-teste, com a aplicação do programa de intervenção REHACOG (Figueira, \& Paixão, in press), e com a utilização dos testes da barragem de 2/3 sinais (Toulouse-Pieron, 1920) e a Versão reduzida das Matrizes Progressivas Coloridas de Raven: IA (Amaral, 1966).

\section{FUNÇÕES E PROCESSOS COGNITIVOS}

Segundo Silva et al. (2006), as funções cognitivas são estruturas básicas que servem de suporte a todas as operações mentais (pensamento, memória, aprendizagem, inteligência, raciocínio, atenção, concentração, tomada de decisões, perceção visual, coordenação motora, etc.). São componentes básicos para a atividade intelectual, com origem nas conexões cerebrais. São capacidades que nos permitem perceber, elaborar e expressar informações. Assim, as funções cognitivas são o esqueleto do pensamento e vão-se estruturando, adaptando e acomodando nos diferentes modos de interação com o ambiente. As operações cognitivas são condutas interiorizadas ou exteriorizadas (um modelo de ação ou um processo de comportamento), pelas quais a pessoa representa os estímulos. Elas são o resultado das nossas capacidades, conforme as necessidades que experimentamos, numa determinada orientação (Silva et al., 2006). A adaptação às exigências diárias é importante para a execução de uma tarefa ou para o delineamento de um objetivo. Neste caso, é necessário planear e criar, com base nas nossas experiências prévias, alternativas capazes de solucionar problemas de modo a serem flexíveis (Ojeda, Peña, Bengoetxea, Segarra, Sánchez, Elizagárate \& Gutiérrez, 2010).

É consensual a pertinência e importância de todos os processos cognitivos, quer nas atividades de vida diária, quer nas académicas. A 
atenção, enquanto processo básico, é uma dos mais analisados e considerados em todos os contextos.

O processo Atenção/Concentração. Sob o ponto de vista etimológico, a atenção provém do termo latino attentionem, que por sua vez deriva de attendere, cujo significado é tender para, debruçar-se sobre. Indica uma ação do sujeito em direção a um objetivo, com vista a ligar-se a ele. A concentração é o vocábulo português para o latino concentrationem, do verbo concentrare, cujo sentido é centrar de todos os lados, confluir. Significa uma focagem precisa do assunto, do objetivo a atingir (Rebelo, 1994). Segundo Vaz (1987), a literatura psicológica sobre dificuldades escolares diferencia-as, com base na autorregulação da vontade. Desatentos seriam os alunos que não querem prestar atenção e desconcentrados aqueles que não conseguem fazer o que lhes é pedido, seja por serem facilmente distraíveis, não conseguindo controlar os seus impulsos, ou por terem grandes dificuldades em mudar de tarefa ou de assunto. É possível verificar que o significado de ambos os termos se relaciona; contudo, cada um deles salienta algumas diferenças. A atenção está mais ligada à primeira reação, reflete mais o estado de alerta; por sua vez, a concentração enfatiza o enfoque, a observação prolongada. A atenção, focagem consistente em algo, tem precisamente esta característica, a de selecionar a informação a ser captada. É o resultado de mecanismos internos existentes no indivíduo, controladores da escolha dos estímulos (Barkley, 1990).

A atenção é um processo cognitivo pelo qual o intelecto focaliza e seleciona estímulos, estabelecendo relação entre eles. A todo instante recebemos estímulos, provenientes das mais diversas fontes; porém só damos atenção a alguns deles, pois não seria possível responder a todos. Além da atenção concentrada, em que se seleciona e processa apenas um estímulo, também pode existir atenção dividida, em que são selecionados e processados diversos estímulos em simultâneo (Mirsy, 1987). A atenção é uma função cognitiva, cerebral, cujo fim é selecionar entre a vaga das estimulações sensoriais, que chegam ao mesmo tempo e incessantemente ao cérebro, as que são úteis e pertinentes para a realização de uma atividade motora ou mental, surgindo como uma propriedade sensorial supramodal do cérebro (Lerner, 1988). Segundo Dember e Warm (1979, in Rebelo, 1994), podemos distinguir na atenção, além do aspeto de 
seleção, as dimensões seguintes: a concentração, que se debruça na tentativa de afastar estímulos interferentes com a noção percetiva; a procura, que corresponde à escolha de alguma característica particular ou de algum grupo específico de entre o conjunto de estímulos; a ativação, que, por sua vez, reflete a prontidão e preocupação com o aparecimento de estímulos; a direção, preparação para a receção de um tipo particular de estímulos e a vigilância ou atenção sustida, que é a capacidade em manter a atenção por períodos prolongados de tempo. Keogh e Margolis (1976) distinguem, na atenção, fases que correspondem sensivelmente às dimensões apresentadas. A primeira, prestar atenção, consiste em ficar num estado de alerta, pôr-se em posição de atendimento; a segunda, focar a atenção, diz respeito a estar vigilante e ter energia suficiente para examinar cuidadosamente os estímulos; a terceira, atenção sustentada, significa concentração, fixação sobre os estímulos durante o tempo necessário e com a atitude adequada para apreendê-los com exatidão.

Ainda, segundo Ojeda et al. (2010) e Alberto (2013), a atenção pode ser sustentada, seletiva, dividida e alternada. Assim, a atenção sustentada, é a habilidade de manter-se focado durante uma atividade contínua e repetitiva, a atenção seletiva é um tipo de atenção consciente, quando escolhemos o foco atencional, a atenção dividida é definida como a capacidade de responder a diferentes estímulos ou tarefas ao mesmo tempo, é uma atenção simultânea que nos permite processar diferentes fontes de informação e executar com êxito mais de uma tarefa ao mesmo tempo e, a atenção alternada, ou seja, atenção focada, consciente, de forma alternada.

Mirsky (1987) propõe um modelo de atenção com base na análise fatorial e identificou os seguintes processos de atenção: a capacidade de focalizar e realizar tarefas durante um curto espaço de tempo; a capacidade de codificar e manipular mentalmente a informação; a capacidade de suster a atenção durante um período mais alargado de tempo e a capacidade de mudar flexivelmente o enfoque da atenção.

Os alunos com défices de atenção têm grandes dificuldades em concretizar estas tarefas. Distraem-se com estímulos irrelevantes, têm problemas na organização dos estímulos e não mantêm dirigida a atenção durante o tempo suficiente para captar corretamente os estímulos (Lerner, 1988). 
Se pensarmos nos fatores potenciadores ou inibidores da atenção/concentração, vemos que o aumento, a diminuição e o desaparecimento da atenção dependem de vários fatores. Os elementos determinantes desta variação costumam ser distinguidos em elementos internos e elementos externos, em relação ao sujeito (Rebelo, 1994). Os elementos interiores dizem respeito às caraterísticas internas do indivíduo, que, em tarefas que requerem atenção e concentração, manifestam variações de desempenho, diferenças que são atribuídas à sua idade, ao seu interesse, à sua motivação, às suas aptidões intelectuais, linguísticas, organizacionais e à sua energia e capacidade de trabalho. Os exteriores referem-se às caraterísticas dos estímulos e das situações com que se confronta diariamente. Assim, os estímulos do meio ambiente despertamlhe mais ou menos atenção em conformidade com as suas variações, características físicas como a grandeza, a intensidade, e o seu movimento; caraterísticas coletivas, o modo como o sujeito os perceciona e o impacto que têm sobre ele ao confrontar-se com eles como a novidade, a surpresa, a incongruência e a sua complexidade.

Segundo Dember e Warm (1979, in Rebelo, 1994), na determinação da atenção parece ser igualmente importante saber como é que o indivíduo reage aos estímulos, isto é, observar as suas reações físicas e as suas atitudes perante os estímulos. Na variação destas reações, o papel mais determinante é geralmente atribuído aos elementos seguintes: a novidade, o inesperado, a intensidade dos estímulos e a habituação a eles. A atenção não é vista como um processo isolado, mas sim como um mecanismo regulador do processamento de informação. Embora certos teóricos defendam a existência de um défice primário de atenção subjacente a esta perturbação, segundo Douglas (1989, in Fonseca, 1998), esta incapacidade de manter a atenção está associada a um défice de autorregulação, embora seja claro que as capacidades de processamento de informação se mantêm intactas. Estes indivíduos apresentam dificuldades em manter a concentração e dificuldades na captação de informação, apresentando um desempenho bastante frágil em tarefas que lhe despertam pouco interesse ou associadas a diversos fatores que facilitam uma maior distração, como é o caso de situações que requerem atenção por longos períodos de tempo, a realização de tarefas repetitivas ou quando existem ruídos (Villar, 1998). Quando interrompidas por outros 
estímulos, apresentam uma maior lentidão em retomar a tarefa, esquecendo-se facilmente das tarefas propostas ou exigidas (APA, 2002). Por consequência, tendem a cometer mais erros ortográficos (Fonseca, 1998). Este tipo de sujeitos também são afetados no que se refere às relações sociais, uma vez que a atenção que prestam nas conversas com os outros é mínima, e mudam frequentemente de assunto, não conseguindo, igualmente, cumprir regras nas atividades lúdicas (APA, 2002).

No que diz respeito à avaliação psicológica dos défices de atenção, esta surge associada a uma perturbação de Hiperatividade, tratando-se assim de uma Perturbação de Hiperatividade com Défice de Atenção (APA, 2014). A característica essencial de Perturbação de Hiperatividade com Défices de Atenção é um padrão persistente de falta de atenção e/ou impulsividade-hiperatividade, com uma intensidade que é mais frequente e grave que o observado habitualmente nos sujeitos com um nível semelhante de desenvolvimento (critério A). O seu diagnóstico atende a mais critérios definidos pela APA (2000), sendo eles: alguns dos sintomas de hiperatividade-impulsividade ou falta de atenção que causam problemas devem ter-se manifestado antes dos 7 anos de idade; contudo, muitos sujeitos são diagnosticados depois de os sintomas terem estado presentes durante vários anos, especialmente no caso de indivíduos com Tipo Predominante Desatento (critério B); alguns problemas relacionados com os sintomas devem ocorrer, pelo menos, em duas situações (por exemplo, em casa, na escola ou no trabalho) (critério C); devem existir provas claras de um défice clinicamente significativo do funcionamento social, académico ou laboral (critério D) e, por fim, a perturbação não ocorre exclusivamente noutro tipo de Perturbações Globais do Desenvolvimento, Esquizofrenia ou outra Perturbação Psicótica que não é melhor explicada por outra perturbação mental. A Perturbação de Hiperatividade com Défices de Atenção apresenta subtipos que derivam do predomínio de certos sintomas (tipo predominantemente desatento, tipo predominantemente hiperativo-impulsivo e tipo misto).

Quanto às estratégias de Intervenção. Embora processo complexo, a atenção é passível de desenvolvimento, muito por via do treino específico, salvaguardando, eventualmente, outras dimensões, como as sensoriais e emocionais ou motivacionais. Assim, por exemplo, para o 
aluno receber a informação que na sala de aula lhe é oferecida do exterior, geralmente pela comunicação do seu professor, é preciso que os seus sentidos se orientem para a fonte da estimulação e que não haja da parte dele impedimentos dessa receção (Rebelo, 1994).

Em primeiro lugar, é necessário que o professor verifique e ateste se os alunos estão a olhar e a escutar. Esta fase designa-se por ativação reticular, estádio percetivo indicador de alguma excitação e alerta por parte do sujeito que aprende (Gagné, 1977). O atendimento é favorecido pelo aumento da intensidade dos estímulos e por mudanças repentinas de estimulação; a ilustração desta variação diz respeito às mudanças de voz do professor, a acentuação que põe em certas palavras ou expressões, a alternância de estímulos auditivos para visuais e vice-versa, o uso de cores e a diminuição ou aumento da luminosidade da sala de aula. Na fase seguinte, a de perceção seletiva, o aluno poderá ser ajudado a concentrarse através duma ativação provocada pelo professor, através de algumas instruções verbais, como sublinhar, colorir e resumir, servindo assim de base para diferenciar os estímulos principais dos secundários. Nesta tarefa os fatores internos do sujeito, o seu desenvolvimento global, o interesse e a motivação desempenham um papel muito relevante. A falta de atenção, sobretudo na sala de aula, pode estar relacionada com a inadaptação dos conteúdos programáticos às suas características (idade, meio familiar e social, atrasos, deficiências) ou a diversos aspetos exteriores ao sujeito. Tudo isto facilita ou dificulta a atenção e a concentração, refletindo-se na motivação e no interesse que o aluno tem pelo assunto e nos resultados da sua aprendizagem. Os alunos com dificuldades de aprendizagem têm frequentemente problemas em organizar a informação que lhes é fornecida e necessitam de aprender formas de o fazer. Assim, Taylor (1964, in Rebelo, 1994) propõe que os alunos organizem a informação, em conformidade com o valor que lhe atribuem, começando pelos conceitos principais e passando a distinguir detalhes em que se apoiam, fazendo a separação de informação relevante da menos importante. Outro processo é fomentar a comparação entre diversas informações, encontrando semelhanças e diferenças, o que leva ao relacionamento entre factos, e à descoberta de relações no que não se tinha pensado anteriormente, ou seja, categorizar informação. 
Outro processo de atenção e concentração passa por ensinar os alunos a investigar, a fazer perguntas, a questionar os assuntos, pedindo-lhes a sua opinião e introduzindo momentos apropriados para a discussão. As estratégias que os alunos com défices de atenção necessitam de aprender são as que fomentam a memorização, como a repetição interior ou a revisão, imagens visuais, a utilização de associações e a tomada de notas. Um fator igualmente importante é a comunicação não-verbal, da parte do professor e dos alunos. Ela é relevante para a atenção e a concentração dos alunos, para a sua autoestima e educação em geral. Através dela é possível verificar o interesse dos alunos, o empenho do professor, os reforços e o feedback; este tipo de comunicação deve ser cuidado no sentido de a conduzir para a sua função de meio facilitador do ensino (Rebelo, 1994).

\section{MÉTODO}

Foi realizado um estudo descritivo e exploratório, com desenho pré e pós-teste, num grupo acessível ${ }^{3}$ de crianças e adolescentes, com sinalização, pelos professores e/ou encarregados de educação, de fragilidades a nível atencional, pela implementação de módulos do programa REHACOG (Figueira \& Paixão, in press) .

Os objetivos são: (i) explorar as potencialidades de alguns módulos do programa de intervenção neuropsicológica REHACOG (Figueira \& Paixão, in press), em crianças com fragilidades de atenção e concentração; (ii) observar a relação entre idade e grau de dificuldades na concretização de algumas tarefas de atenção do REHACOG (Figueira \& Paixão, in press).

Procedimentos. Num primeiro momento, foram aplicados dois testes que avaliam a atenção, o IA-versão reduzida das Matrizes Progressivas Coloridas de Raven (Amaral, 1966) e o teste da barragem 2/3 sinais (Toulouse-Pieron, 1920). Num segundo momento, foram utilizadas, como treino/intervenção, tarefas do módulo atenção, do programa REHACOG (Figueira \& Paixão, in press), que dispõe de algumas tarefas de treino,

\footnotetext{
${ }^{3}$ A amostra foi reunida no Centro de Estudos, StudyHelp, em Pevidém-Guimarães, onde nos foi pertinente e acessível realizar esta investigação, devido à evidência de dificuldades ao nível da atenção e por conhecimento prévio do grupo de participantes.
} 
intervindo em quatro níveis de atenção: a atenção sustentada, a atenção seletiva, a atenção dividida e a atenção alternada.

Após a aplicação de algumas tarefas do módulo de atenção do programa de intervenção, foram aplicados novamente os testes de atenção anteriormente referidos. Por uma questão de gestão de tempo, alguns testes foram aplicados de forma individual, e outros de forma conjunta, dispostos no máximo por 4 elementos numa sala. Foram enviados os pedidos de autorização e consentimento informado aos pais, garantindo, assim, as questões éticas e deontológicas.

Participantes. O grupo é composto por $37 \quad(\mathrm{~N}=37)$ crianças/adolescentes com sinalização, por parte dos professores e/ou encarregados de educação, de dificuldades ao nível da atenção e concentração, dos 7 aos 17 anos, com frequência escolar, que varia entre o $1^{\circ}$ e o $9^{\circ}$ ano de escolaridade. O primeiro ciclo $\left(1^{\circ}\right.$ ao $4^{\circ}$ ano de escolaridade) é constituído por 14 crianças, dos 7 aos 10 anos de idade, em que 7 são do sexo feminino e 7 do sexo masculino; o segundo ciclo $\left(5^{\circ}\right.$ e $6^{\circ}$ ano de escolaridade) é composto por 10 crianças e 2 adolescentes, dos 10 aos 15 anos de idade, em que 5 são do sexo feminino e 7 são do sexo masculino. Por fim, no terceiro ciclo ( $7^{\circ}$ ao $9^{\circ}$ ano de escolaridade), existem 11 crianças/adolescentes dos 12 aos 17 anos de idade, em que 6 são do sexo feminino e 5 são do sexo masculino. No que diz respeito ao total, ao nível do género (19 rapazes e 18 raparigas) verifica-se uma diferença mínima no que diz respeito à percentagem de crianças em ambos os géneros.

Instrumentos. Para a recolha dos dados e de forma a avaliar as crianças e os adolescentes que constituem o grupo da amostra deste estudo, foram utilizados os seguintes instrumentos:

Teste da Barragem de 2/3 sinais (Toulouse-Pieron, 1920): Este teste avalia, essencialmente, a atenção seletiva e a sustentada. Adicionalmente, são necessárias capacidades de exploração visual com exatidão, ativação e inibição de respostas rápidas. $\mathrm{O}$ instrumento consiste em duas folhas A4, com 1600 quadrados, dos quais, apenas 10 ou 15 (consoante se trate da Barragem de 2 ou 3 sinais) em cada linha são iguais a um dos modelos apresentados no topo da folha. A tarefa do sujeito consiste em desenhar um traço por cima dos quadrados alvo, dispondo de até 10 minutos. 
Quanto às pontuações, podemos encontrar o número de estímulos corretos (C), que se refere ao número de estímulos que o sujeito acertou, as lacunas (L), ou seja, os estímulos que o sujeito omitiu e os erros (E), ou os estímulos que assinalou incorretamente. $\mathrm{O}$ resultado total é calculado a partir da fórmula $[\mathrm{C}-(\mathrm{L}+\mathrm{E} \times 2+1)] / 10$ ou, na ausência de lacunas e erros, por $\mathrm{C} / 10$, em que o resultado que advém do cálculo da fórmula referida acima resulta numa média que será analisada em consonância com os dados normativos da população alvo.

IA (Amaral, 1966), versão reduzida das Matrizes Progressivas Coloridas de Raven - O Teste das Matrizes Progressivas Coloridas de Raven (MPCR) consiste numa prova de inteligência que existe disponível em três versões diferentes: a forma Geral (Standard Progressive Matrices - SPM) constituída por 60 itens (12 em cada série - A, B, C, D e E), a forma Especial ou Colorida (Coloured Progressive Matrices - CPM), com 36 itens (12 em cada série - A e B) e a forma Avançada (Advanced Progressive Matrices - APM), composta por 48 itens (12 numa série Set I e 36 noutra - Set II) (Simões, 1994). O termo progressivo está relacionado com o facto de o nível de dificuldade ir aumentando ao longo do teste, não só de item para item como também de série para série (Simões, 2000). Essencialmente, o Teste das Matrizes de Raven (19381962) compõe-se de um conjunto de tarefas não-verbais, com o objetivo de medir a aptidão e atenção do sujeito para apreender relações entre figuras. A pessoa necessita apenas de indicar/apontar a figura que considera resolver o problema apresentado (Raven, Court \& Raven, 1998). Todos os itens do teste foram pensados de modo a que a sua solução ocorra do ponto de vista percetivo, espacial ou lógico de uma configuração (gestalt) (Pascuali, Wechsler \& Bensusan, 2002). Cada item pode constituir um problema cuja resposta se desenvolve através de um processo de compreensão e que termina com um processo de solução. Os problemas presentes nas MPCR correspondem, na terminologia de Greeno (1978, in Simões, 1994), a problemas do tipo "indução de estrutura", em que a tarefa principal do sujeito consiste em identificar a regra ou padrão estrutural presente. $O$ teste pode ser aplicado individual ou coletivamente. Cada item é cotado com um ponto se o sujeito tiver respondido corretamente e com zero se a resposta dada for incorreta. As MPCR são um dos poucos testes adequadamente projetados para poderem 
ser aplicáveis a um vasto conjunto de sujeitos, quer no que diz respeito à idade (da infância à velhice), quer no que diz respeito à aptidão (Simões, 1994). Em 1966, foi editada em Portugal uma versão reduzida do teste das MPCR: o IA. Este teste é constituído por 30 itens. REHACOG, Programa de intervenção/promoção cognitiva e emocional (Figueira \& Paixão, in press): O REHACOG (Reabilitação Cognitiva) é uma adaptação do REHACOP (Reabilitação Cognitiva em Psicoses) (2012) que, originalmente, se assume como um programa de reabilitação neuropsicológica de psicoses e esquizofrenia (Ojeda, Peña, Bengoetxea, Segarra, Sánchez, Elizagárate, \& Gutiérrez, 2010). O programa de intervenção resulta de um longo trabalho, de vários anos, de vários especialistas em neuropsicologia, com mais de 18 anos de experiência na área, e várias vezes premiado. Todavia, neste momento, os autores da versão original, espanhola, têm projetos de utilização com outras populações. O mesmo estará a ser tentado em Portugal. Trata-se de um programa integral e estruturado, constituído por 8 módulos de intervenção: atenção, linguagem, memória, funções executivas, atividades da vida diária, competências sociais, cognição social e psicoeducação (Figueira \& Paixão, in press). É composto por um total de 300 exercícios de intervenção, acessíveis de administração, com instruções, soluções e orientações para avaliação das mudanças, fichas de seguimento e sugestões de seguimento, de tarefas para realizar em contexto familiar. Inclui recomendações e orientações sobre como avaliar as alterações ocorridas ou ganhos adquiridos. Os materiais são acessíveis e coloridos e incluem as instruções para os pacientes e as soluções para o terapeuta. Pode ser utilizado em sessões individuais ou em grupo. $\mathrm{O}$ programa completo é composto por dois manuais, um manual para o terapeuta e um manual para o paciente (Figueira \& Paixão, in press).

Nesta investigação foram aplicadas apenas algumas tarefas do módulo atenção (composto por 4 submódulos: atenção sustentada, atenção dividida, atenção alternada e atenção seletiva). Na atenção sustentada foram aplicadas as tarefas: números I, figuras I e verduras, que consistem em tarefas de contagem total de um número, figura e verduras que se encontram dispersos entre outros aleatórios e a tarefa símbolos I, que recorre à identificação de símbolos exatos em cada linha, onde se encontram outros símbolos semelhantes. $\mathrm{Na}$ atenção seletiva, foram 
administrados o cancelamento do número seguinte I, que consiste na seleção do número que vem no seguimento dos números que estão "cancelados" (marcados), a tarefa das imagens sobrepostas que pretende que o sujeito identifique o número de imagens que vê numa só figura, identificando-as, e, por fim, a tarefa das caras onde são apresentadas oito alíneas compostas por quatro rostos, onde o sujeito deve fazer corresponder o rosto "padrão" de cada alínea. Na atenção dividida, foi aplicada a tarefa dos sinais de trânsito, que tem o objetivo de ser uma tarefa de contagem, onde o sujeito deve contabilizar o número de vezes que surge o sinal de trânsito identificado inicialmente. Por fim, na atenção alternada foi aplicada a tarefa dos ícones, onde existem vinte e duas alíneas com várias disposições faciais de bonecos padrões e cujo objetivo consiste na identificação, em cada alínea, do número de bonecos iguais que encontra. Foi contabilizado o número de acertos, com valor de 1 cada.

\section{RESULTADOS}

Para a realização das análises estatísticas dos dados utilizou-se o programa Statistical Package for Social Sciences (SPSS - versão 22). Para a caracterização do perfil da amostra, bem como para exploração dos dados, no que diz respeito ao impacto da aplicação de algumas tarefas do módulo de atenção, do programa de intervenção REHACOG (Figueira \& Paixão, in press), segundo uma metodologia de pré e pós-teste, recorremos às estatísticas descritivas.

Análise das estatísticas descritivas relativas aos resultados pré-e pós-teste nos testes de Atenção (IA e Barragem), por ciclos de escolaridade: De acordo com as estatísticas descritivas (Tabela 1), podemos verificar que no primeiro ciclo de escolaridade, no teste de atenção IA, verificamos um aumento dos resultados do pré $(M=17.214$, $\mathrm{dp}=4.0796)$ para o pós-teste $(\mathrm{M}=18.286, \mathrm{dp}=4.1404)$, sabendo que o mínimo de acertos possível seria 0 pontos e o máximo potencial seria 30 pontos. No teste da Barragem de 2/3 sinais também podemos apurar um aumento da média do pré para o pós-teste (pré-teste $\mathrm{M}=10.650$, $\mathrm{dp}=3.9764$; pós-teste $\mathrm{M}=11.036, \mathrm{dp}=3.7399$ ). No segundo ciclo de escolaridade, ainda quanto aos acertos, também se verifica um aumento 
das médias nos resultados no IA, do pré para o pós-teste (pré-teste $\mathrm{M}=17.500, \mathrm{dp}=3.233$; pós-teste $\mathrm{M}=18.917, \mathrm{dp}=2.7455)$ e na Barragem de $2 / 3$ sinais (pré-teste $\mathrm{M}=17.983, \mathrm{dp}=4.8581$; pós-teste $\mathrm{M}=18.917$, $\mathrm{dp}=4.5405)$. Após a análise dos dados, verificamos que existe uma diminuição na média de erros no IA (pré-teste $\mathrm{M}=12.500$, $\mathrm{dp}=3.2333 \mathrm{e}$ pós-teste $\mathrm{M}=11.083, \mathrm{dp}=2.7455)$. No terceiro ciclo de escolaridade, podemos apurar que existe um aumento mais significativo das médias, em comparação com os ciclos anteriores, no teste IA (pré-teste $M=19.364$, $\mathrm{dp}=3.9818$; pós-teste $\mathrm{M}=21.273$, $\mathrm{dp}=3.3494$ ) e no teste da Barragem (préteste $\mathrm{M}=15.273 \mathrm{dp}=4.0455$ e pós-teste $\mathrm{M}=16.709$ e $\mathrm{dp}=4.9387$ ), existindo também uma diminuição na média de erros no teste IA (pré-teste $\mathrm{M}=10.636, \mathrm{dp}=3.9818$ e pós-teste $\mathrm{M}=8.727, \mathrm{dp}=3.3494$ ).

\section{Tabela 1}

Médias e desvios-padrão dos resultados da aplicação dos testes de atenção (IA e Barragem) pré-teste e pós-teste, por ciclos de escolaridade

\begin{tabular}{lllllll}
\hline \multirow{2}{*}{ Escolaridade } & Erros/Acertos & $\mathrm{N}$ & Média & $\begin{array}{l}\text { Desvio- } \\
\text { Padrão }\end{array}$ & Mínimo & Máximo \\
\hline $1^{\circ}$ ciclo & IA_Acertos_Pré & 14 & 17.214 & 4.0796 & 9.0 & 24.0 \\
& IA_Erros_Pré & 14 & 12.786 & 4.0796 & 6.0 & 21.0 \\
& Barragem_Pré & 14 & 10.650 & 3.9764 & 5.6 & 18.9 \\
& IA_Acertos_Pós & 14 & 18.286 & 4.1404 & 10.0 & 26.0 \\
& IA_Erros_Pós & 14 & 11.714 & 4.1404 & 4.0 & 20.0 \\
& Barragem_Pós & 14 & 11.036 & 3.7399 & 6.6 & 18.7 \\
\hline $2^{\circ}$ ciclo & IA_Acertos_Pré & 12 & 17.500 & 3.2333 & 13.0 & 22.0 \\
& IA_Erros_Pré & 12 & 12.500 & 3.2333 & 8.0 & 17.0 \\
& Barragem_Pré & 12 & 17.983 & 4.8581 & 12.8 & 29.6 \\
& IA_Acertos_Pós & 12 & 18.917 & 2.7455 & 15.0 & 24.0 \\
& IA_Erros_Pós & 12 & 11.083 & 2.7455 & 6.0 & 15.0 \\
& Barragem_Pós & 12 & 18.917 & 4.5405 & 14.8 & 29.8 \\
\hline $3^{\circ}$ ciclo & IA_Acertos_Pré & 11 & 19.364 & 3.9818 & 12.0 & 24.0 \\
& IA_Erros_Pré & 11 & 10.636 & 3.9818 & 6.0 & 18.0 \\
& Barragem_Pré & 11 & 15.273 & 4.0455 & 6.6 & 22.6 \\
& IA_Acertos_Pós & 11 & 21.273 & 3.3494 & 15.0 & 25.0 \\
& IA_Erros_Pós & 11 & 8.727 & 3.3494 & 5.0 & 15.0 \\
& Barragem_Pós & 11 & 16.709 & 4.9387 & 5.6 & 23.9 \\
\hline
\end{tabular}

Análise das estatísticas descritivas dos diferentes níveis de escolaridade no REHACOG

Módulo Atenção Dividida: De acordo com os dados da tabela 2, podemos verificar que os resultados da aplicação da tarefa Sinais de 
Trânsito, do módulo Atenção Dividida, encontram-se abaixo da média no $1^{\circ}$ ciclo $(\mathrm{M}=.286, \mathrm{dp}=.4688)$ e no $2^{\circ}$ ciclo $(\mathrm{M}=.250, \mathrm{dp}=.4523)$, visto que a média potencial será de 0.5 , dado as respostas variarem de $0-1$. Ainda, que a média, no $3^{\circ}$ ciclo de escolaridade, encontra-se acima da média face à pontuação máxima possível, numa média potencial de .5 (M=.727, $\mathrm{dp}=.4671$ ). A partir destes dados podemos inferir a possibilidade do grau de dificuldade da tarefa Sinais de Trânsito ser bastante elevado para crianças no $1^{\circ}$ ciclo e $2^{\circ}$ ciclo de escolaridade com fragilidades ao nível da atenção.

\section{Tabela 2}

Médias e Desvios Padrão, dos diferentes níveis de escolaridade, face às pontuações da aplicação do programa de intervenção REHACOG, da tarefa do módulo Atenção Dividida

\begin{tabular}{lll}
\hline & & $\begin{array}{l}\text { Sinais de Trânsito } \\
\text { Acertos }(0-1)\end{array}$ \\
\hline $1^{\circ}$ ciclo & $\mathrm{N}$ & 14 \\
& Média & .286 \\
& Desvio-Padrão & .4688 \\
\hline $2^{\circ}$ ciclo & $\mathrm{N}$ & 12 \\
& Média & .250 \\
& Desvio-Padrão & .4523 \\
\hline $3^{\circ}$ ciclo & $\mathrm{N}$ & 11 \\
& Média & .727 \\
& Desvio-Padrão & .4671 \\
\hline
\end{tabular}

Módulo Atenção Alternada: Através da tabela 3, podemos verificar que os resultados obtidos da aplicação da tarefa Ícones do módulo Atenção Alternada se encontram ligeiramente abaixo da média no $2^{\circ}$ ciclo de estudos $(\mathrm{M}=10.083, \mathrm{dp}=2.3916)$ e no $3^{\circ}$ ciclo de estudos $(\mathrm{M}=10.091$, $\mathrm{dp}=2.0226$ ), em que o potencial de respostas nesta tarefa seria no mínimo 0 e no máximo 22 (com média potencial de 11). No primeiro ciclo, a média de respostas encontra-se bastante abaixo da média $(M=7.429, \mathrm{dp}=3.8772)$. Podemos afirmar que os resultados desta tarefa encontram-se abaixo da média potencial de respostas, possivelmente devido a alguma saturação sentida durante a execução da tarefa, em que a tarefa integra vinte e duas alíneas com vários disposições faciais de bonecos padrões e o objetivo é o sujeito identificar, em cada alínea, quantos bonecos iguais encontra, 
tratando-se de um exercício de atenção a ser adaptado às diferentes idades e características do grupo-alvo.

Tabela 3.

Médias e Desvios Padrão, dos diferentes níveis de escolaridade, face às pontuações da aplicação do programa de intervenção REHACOG, da tarefa do módulo Atenção

Alternada

\begin{tabular}{lll}
\hline & & $\begin{array}{l}\text { Ícones } \\
(0-22)\end{array}$ \\
\hline $1^{\circ}$ ciclo & $\mathrm{N}$ & 14 \\
& Média & 7.429 \\
& Desvio-Padrão & 3.8772 \\
\hline $2^{\circ}$ ciclo & $\mathrm{N}$ & 12 \\
& Média & 10.083 \\
& Desvio-Padrão & 2.3916 \\
\hline $3^{\circ}$ ciclo & $\mathrm{N}$ & 11 \\
& Média & 10.091 \\
& Desvio-Padrão & 2.0226 \\
\hline
\end{tabular}

Módulo Atenção Seletiva: De acordo com os dados da tabela 4, podemos verificar que, de uma forma geral, no módulo Atenção Seletiva, a tarefa que corresponde aos dados menos satisfatórios, em todos os ciclos de escolaridade, é a tarefa Cancelamento do Número Seguinte 1, que corresponde a uma tarefa que exige bastante atenção na sua execução. Assim, temos no primeiro ciclo $(\mathrm{M}=.143, \mathrm{dp}=.3631)$ e no segundo ciclo $(\mathrm{M}=.417, \mathrm{dp}=.5149)$, que correspondem a valores abaixo do esperado dentro dos valores possíveis entre 0 e 1 (com média potencial de 0.5 ). No terceiro ciclo verificam-se médias mais elevadas $(\mathrm{M}=.727, \mathrm{dp}=.4671)$, resultados que se encontram acima do valor médio (.5). Nas tarefas Caras e Imagens Sobrepostas, verificamos que todos os ciclos se encontram acima da média de acertos possível, entre 0 e 8 (Caras) e 0-18 (Imagens Sobrepostas).

Relativamente à tarefa Caras, no primeiro ciclo de escolaridade $(\mathrm{M}=5.643$ e $\mathrm{dp}=2.6197)$, no segundo ciclo $(\mathrm{M}=5.417, \mathrm{dp}=2.6443)$ e no terceiro ciclo $(\mathrm{M}=7.455, \mathrm{dp}=.6876)$, verifica-se uma média de acertos superior, em relação à média potencial (mínimo de acertos 0 e máximo de acertos possível 8). Por fim, na tarefa Imagens Sobrepostas no primeiro ciclo verificamos uma média de acertos $(\mathrm{M}=16.857, \mathrm{dp}=1.3506)$, no segundo ciclo $(\mathrm{M}=17.583, \mathrm{dp}=.6686)$ e no terceiro ciclo (17.636), 
apuramos uma média de acertos superior, em relação à média potencial (mínimo de acertos 0 e máximo 18).

\section{Tabela 4}

Médias e Desvios Padrão, dos diferentes níveis de escolaridade, face às pontuações da aplicação do programa de intervenção REHACOG, das tarefas do módulo Atenção Seletiva

\begin{tabular}{ccccc}
\hline Escolaridade & & $\begin{array}{c}\text { CNS1 } \\
\text { Acertos (0-1) }\end{array}$ & $\begin{array}{c}\text { Caras } \\
\text { Acertos (0-8) }\end{array}$ & $\begin{array}{c}\text { Imagens Sobrepostas } \\
\text { Acertos (0-18) }\end{array}$ \\
\hline $1^{\circ}$ ciclo & $\mathrm{N}$ & 14 & 14 & 14 \\
& Média & .143 & 5.6 & 16.857 \\
& & 43 & 1.3506 \\
& Desvio- & .3631 & 2.6 & 12 \\
& Padrão & & 197 & 17.583 \\
\hline $2^{\circ}$ ciclo & $\mathrm{N}$ & 12 & 12 & .6686 \\
& Média & .417 & 5.4 & 11 \\
& & & 17 & 17.636 \\
& Desvio- & .5149 & 2.6 & .5045 \\
& Padrão & & 443 & \\
\hline $3^{\circ}$ ciclo & $\mathrm{N}$ & 11 & 11 & 7.4 \\
& Média & .727 & 55 & .68 \\
& & 76 &
\end{tabular}

(CNS1 - Cancelamento do número seguinte 1)

Módulo Atenção Sustentada: De acordo com as estatísticas descritivas e análise exploratória, na tabela 5, podemos verificar que, relativamente à primeira tarefa, Números1, do módulo atenção sustentada, existem médias muito baixas. No primeiro, segundo e terceiro ciclos $(\mathrm{M}=.000, \mathrm{dp}=.0000)$ não existem respostas certas, o que nos remete para o total insucesso da tarefa nesta amostra. Nas restantes tarefas do módulo atenção sustentada, encontram-se valores positivos relativamente ao potencial esperado de cada exercício. 


\section{Ana Paula Couceiro Figueira e Filipa Rocha}

\section{Tabela 5}

Médias e Desvios Padrão, dos diferentes graus de escolaridade, face as pontuações da aplicação do programa de intervenção REHACOG, das tarefas do módulo

Atenção Sustentada

\begin{tabular}{lcllll}
\hline & & $\begin{array}{l}\text { NúmerosI } \\
\text { Acertos (0-1) }\end{array}$ & $\begin{array}{l}\text { FigurasI } \\
\text { Acertos (0-1) }\end{array}$ & $\begin{array}{l}\text { SímbolosI } \\
\text { Acertos (0-17) }\end{array}$ & $\begin{array}{l}\text { Verduras } \\
\text { Acertos (0-1) }\end{array}$ \\
\hline $1^{\circ}$ ciclo & $\mathrm{N}$ & 14 & 14 & 14 & 14 \\
& Média & .000 & .286 & 15.643 & .214 \\
& Desvio- & .0000 & .4688 & 1.5495 & .4258 \\
& Padrão & & & & \\
\hline $2^{\circ}$ ciclo & $\mathrm{N}$ & 12 & 12 & 12 & 12 \\
& Média & .000 & .500 & 16.500 & .500 \\
& Desvio- & .0000 & .5222 & .6742 & .5222 \\
& Padrão & & & & \\
\hline $3^{\circ}$ ciclo & $\mathrm{N}$ & 11 & 11 & 11 & 11 \\
& Média & .000 & .636 & 16.545 & .909 \\
& Desvio- & .0000 & .5045 & .5222 & .3015 \\
& Padrão & & & & \\
\hline
\end{tabular}

Assim, na tarefa Figuras 1, com acertos que variam entre 0 e 1, no primeiro ciclo não se verificam valores acima da média $(\mathrm{M}=.286$, $\mathrm{dp}=.4688$ ), mas nos restantes ciclos, o número de acertos apresenta valores ligeiramente acima (primeiro ciclo: $\mathrm{M}=.500, \mathrm{dp}=.5222$; segundo ciclo: $\mathrm{M}=.636, \mathrm{dp}=.5045)$. Na tarefa Símbolos 1 , com acertos possíveis entre os 0 e 17, no primeiro ciclo encontramos $(\mathrm{M}=15.643$, $\mathrm{dp}=1.5495)$, o segundo ciclo $(\mathrm{M}=16.500, \mathrm{dp}=.6742)$ e o terceiro ciclo apresenta uma média de acertos muito alta, relativamente ao potencial de acertos desta tarefa $(\mathrm{M}=16.545, \mathrm{dp}=.5222)$. Relativamente à última tarefa deste módulo, Verduras, a média de acertos (que varia entre 0 e 1) também se apresenta com uma média de acertos positiva no terceiro ciclo $(\mathrm{M}=.909$, $\mathrm{dp}=.3015)$, havendo uma aproximação da média de acertos à média de acertos total (1). No segundo ciclo, a média apresentada é igualmente positiva $(\mathrm{M}=.500, \mathrm{dp}=.5222)$, sendo que o primeiro ciclo apresenta valores abaixo da média $(\mathrm{M}=.214, \mathrm{dp}=.4258)$, revelando dificuldades de execução.

\section{CONCLUSÕES}

Com esta investigação pretendeu-se explorar os ganhos, ao nível da atenção, face à aplicação de algumas tarefas do módulo atenção do 
programa de intervenção REHACOG, sendo um estudo descritivo e exploratório com crianças e adolescentes com fragilidades atencionais. Neste trabalho foram formulados como objetivos: verificar o impacto do programa REHACOG em crianças com fragilidades de atenção e concentração; ver a relação entre idade e grau de dificuldades na concretização de algumas tarefas de atenção do REHACOG. Embora existam diferenças no pré-teste em relação aos testes de atenção, intergrupos de escolaridade, não se pode atribuir à intervenção específica com as tarefas do programa de REHACOG. De facto, o plano utilizado não nos permite realizar este tipo de inferência. Contudo, pelas realizações das tarefas do próprio programa, podemos aventar da sua utilidade na potenciação da atenção dos sujeitos. Existem outras hipóteses explicativas para os ganhos no pós-teste, desde aprendizagem dos testes de atenção da Barragem de $2 / 3$ sinais e do IA, maturidade, desenvolvimento, aprendizagem, estímulos escolares e outras.

A partir dos resultados, consideramos que o programa de intervenção REHACOG se revela útil e mais acessível para crianças/adolescentes que frequentam o $2^{\circ}$ e o $3^{\circ}$ ciclo de escolaridade. Foi possível verificar, por vezes, a saturação das crianças do $1^{\circ}$ ciclo, durante a realização das tarefas do programa REHACOG, acabando muitas vezes por não ser concluída a tarefa, ou seja, os exercícios revelaram-se difíceis para esta faixa etária. Como em qualquer investigação de natureza científica, este estudo apresenta algumas limitações. O tamanho da amostra utilizada é relativamente pequeno $(\mathrm{N}=37)$, embora este estudo seja apenas exploratório numa amostra que nos foi acessível. Seria necessário e importante recorrer a amostras mais alargadas e heterogéneas, bem como utilizar procedimentos de amostragem mais robustos. Igualmente, no sentido de testar a capacidade e o poder de intervenção do REHACOG, deveríamos ter acesso a uma amostra mais robusta para possibilitar a realização de um plano experimental ou de um plano quasi-experimental.

\section{REFERÊNCIAS BIBLIOGRÁFICAS}

Alberto, I. (2003). Atenção, por favor (!) à avaliação da atenção! Psychologica, 34(3), 231-244. 
Amaral, J. R. (1966). Teste IA. Aferiçao do teste I.A.: escala reduzida das matrizes progressivas de J. C. Raven. Lisboa: Fundação Calouste Gulbenkian.

American Psychiatric Association (2002). DSM-IV-TR- Manual de Diagnóstico e Estatística das Perturbações Mentais. Lisboa: Climepsi Editores.

American Psychiatric Association (2014). DSM-V - Manual diagnóstico e estatístico de transtornos mentais. Porto Alegre: Artmed Editores.

Barkley, R. (1990). Attention deficit hyperactivity disorder: A handbook for diagnosis and treatment. New York: The Guilford Press.

Figueira, A. P. \& Paixão, R. (in press). REHACOG - Programa de intervenção/promoção cognitiva.

Fonseca, A. C., Simões, M. R., Fernandes, J. R., et al. (1998). Hiperactividade na comunidade e hiperactividade em meio clínico: semelhanças e diferenças. Psychologica, 19(2), 27-56.

Karande, S. \& Kulkarni, M. (2005). Poor school performance. Indian Journal of Pediatrics, 72(11), 961-967.

Keogh, B. \& Margolis, J. (1976). A Component Analysis of Attentional Problems of Educationally Handicapped Boys. Journal of Abnormal Child Psychology, 4(4), 349-359.

Lerner, J. (1988). Learning disabilities: Theories, diagnosis and teaching strategies. Boston: Houghton Mifflin Comp.

Mirsky, A. (1987). Behavioral and psycholophysiological markers of disordered attention. Environmental Health Perspectives, 74(2), 191199.

Ojeda, N., Peña, J., Bengoetxea, E., Segarra, R., Sánchez, P., Elizagárate, E., \& Gutiérrez, M. (2010). Cognitive rehabilitation in executive functioning and processing speed in schizophrenia and first-episode psychosis. European Psychiatry, 25(supl 1), 1102-1121.

Raven, J. C., Court, J. H. \& Raven, J. (1998). Manual for Raven's Progressive Matrices and Vocabulary Scales Section: Introducing parallel versions of the CPM and SPM together with a more powerfull version of the SPM (SPM-Plus). Oxford: Oxford Psychologists Press. Rebelo, J. A. (1994). Estratégias para a Concentração. Revista Portuguesa de Pedagogia, 2(3), 171-182. 
Seligman, M. (2002). Positive psychology, positive prevention, and positive therapy. In C. Snyder, \& S. Lopez (Orgs.), The handbook of positive psychology (pp. 3-12). New York: Oxford Press.

Silva, I., Pais-Ribeiro, J., \& Cardoso, H. (2006). Alterações das Funções Cognitivas em indivíduos com diabetes mellitus: a importância das características demográficas e clínicas. Revista Referencia, 2(2), 3341.

Simões, M. R. (1994). Investigações no âmbito da aferição nacional do Teste das Matrizes Progressivas Coloridas de Raven (M.P.C.R.). Dissertação de Doutoramento em Psicologia, especialização em Avaliação Psicológica, apresentada à Faculdade de Psicologia e de Ciências da Educação da Universidade de Coimbra

Simões, M. R. (2000). Investigações no âmbito da aferição nacional do Teste das Matrizes Progressivas Coloridas de Raven (M.P.C.R.). Lisboa: Fundação Calouste Gulbenkian.

Vaz, J. L. P. (1987). As capacidades de mobilização do aluno desatento. (Dissertação de Mestrado). Aveiro: Universidade de Aveiro.

Villar, I. (1998). Déficit de Atención con Hiperactividad: Manual para Padres y Educadores. Madrid: Editorial:Cepev. 